\title{
Scan-rescan reproducibility of diastolic left ventricular kinetic energy, viscous energy loss and vorticity assessment using 4D flow MRI: analysis in healthy subjects
}

\author{
Vivian P. Kamphuis ${ }^{1,2}$. Jos J. M. Westenberg ${ }^{3} \cdot$ Roel L. F. van der Palen ${ }^{1} \cdot$ Pieter J. van den Boogaard ${ }^{3}$. \\ Rob J. van der Geest ${ }^{3} \cdot$ Albert de Roos ${ }^{3} \cdot$ Nico A. Blom ${ }^{1,4} \cdot$ Arno A. W. Roest ${ }^{1} \cdot$ Mohammed S. M. Elbaz $^{3}$
}

Received: 5 October 2017 / Accepted: 19 December 2017 / Published online: 5 January 2018

(c) The Author(s) 2017. This article is an open access publication

\begin{abstract}
The aim of the current study was to assess the scan-rescan reproducibility of left ventricular (LV) kinetic energy (KE), viscous energy loss (EL) and vorticity during diastole from four-dimensional flow magnetic resonance imaging (4D flow MRI) in healthy subjects. Twelve volunteers (age $27 \pm 3$ years) underwent whole-heart 4D flow MRI twice in one session. In-scan consistency was evaluated by correlation between $\mathrm{KE}$ and $\mathrm{EL} . \mathrm{EL}_{\text {index }}$ was computed to measure the amount of EL relative to KE over diastole. Scan-rescan analysis was performed to test reproducibility of volumetric measurements of KE, $\mathrm{EL}, \mathrm{EL}_{\text {index }}$ and vorticity in the LV over early (E) and late (A) diastolic filling. In-scan consistency between KE and EL was strong-excellent (E-filling scan1: $\mathrm{r}=0.92, P<0.001$; scan2: $\rho=0.96, P<0.001$ and A-filling scan1: $\rho=0.87, P<0.001$; scan2: $\mathrm{r}=0.99, P<0.001$ ). For the majority of subjects (10 out of 12$)$, KE and EL measures showed good to strong reproducibility. However, with a wide range of agreement [intraclass correlation (ICC): 0.64-0.95] and coefficients of variation (CV) $\leq 25 \%$. $\mathrm{EL}_{\text {index }}$ showed strong reproducibility for all 12 subjects with a strong ICC $(0.94, P<0.001)$ and a CV of $9 \%$. Scan-rescan reproducibility of volumetric vorticity showed good-excellent ICCs $(0.83-0.95)$ with CVs $\leq 11 \%$. In conclusion, the current study shows strong-excellent in-scan consistency and overall good agreement between scans for 4D flow MRI assessment of left ventricular kinetic energy, energy loss and vorticity over diastole. However, substantial differences between the scans were also found in some parameters in two out of twelve subjects. Strong reproducibility was found in the dimensionless $\mathrm{EL}_{\text {index }}$, which measures the amount of viscous energy loss relative to the average kinetic energy over diastole.
\end{abstract}

Keywords 4D flow MRI · Magnetic resonance imaging $\cdot$ Kinetic energy $\cdot$ Viscous energy loss $\cdot$ Vorticity

\section{Introduction}

Congenital and acquired heart diseases affect the efficacy of intracardiac flow patterns and energy distribution [1, 2]. Given the three-dimensional (3D) time-varying nature of these flow patterns and energetics, specialized in vivo

Vivian P. Kamphuis

v.p.kamphuis@lumc.nl

1 Division of Pediatric Cardiology, Department of Pediatrics, Leiden University Medical Center, Leiden, The Netherlands

2 Netherlands Heart Institute, Utrecht, The Netherlands

3 Department of Radiology, Leiden University Medical Center, Leiden, The Netherlands

4 Division of Pediatric Cardiology, Department of Pediatrics, Academic Medical Center, Amsterdam, The Netherlands imaging and analysis techniques are needed to evaluate volumetric changes in such complex hemodynamic parameters. Four-dimensional flow magnetic resonance imaging (4D flow MRI) allows for comprehensive non-invasive assessment of 3D time-varying blood flow properties in the heart and great vessels in all three velocity encoding directions and spatial dimensions over the cardiac cycle [3].

4D flow MRI has recently emerged as a novel tool for in vivo quantification of intracardiac flow energetics, associated energy losses and vortical flow patterns by means of kinetic energy (KE) [4-7], viscous energy loss (EL) [1] and vorticity [8,9]. KE is the energy contained in the flow of the bloodstream due to motion and EL is the KE that is irreversibly lost due to viscosity-induced frictional forces within the blood flow [1]. In acquired heart disease, remodelling occurs which can lead to alterations in intracardiac hemodynamics [4]. Alteration in 
intraventricular KE derived from 4D flow MRI has been used to assess left ventricular (LV) and right ventricular (RV) (dys)function in patients with different stages of heart failure (HF) [4-7]. This is also the case in various congenital heart diseases. Even after correction, patients may develop systolic and/or diastolic dysfunction, leading to changes in intracardiac flow energetics [1]. In addition, intracardiac anatomy may not be restored. In corrected atrioventricular septal defect patients, elevated EL was associated with altered 3D vortex ring formation in the LV filling pattern [1]. Vorticity, the curl of velocity, is a fundamental quantity in fluid mechanics that describes the local spinning rate of fluid particles and can characterize vortex flow [10]. Quantitative vortex parameters, such as vorticity, have been used to assess diastolic (dys) function in several patient groups $[8,9,11,12]$. Furthermore, in patients with complex congenital intracardiac deformations such as after the Fontan operation, flow collision with remaining septal structures and stagnation of flow through a ventricular septal defect may result in altered EL and vortex formation [13].

Recently, good reproducibility of inflow- and outflowassessment from 4D flow MRI was shown [14]. However, there is a lack of studies validating the reproducibility of intracardiac energy and quantitative vorticity parameters from 4D flow MRI in a scan-rescan setting. Scan-rescan reproducibility is important for clinical applicability as it expresses the reliability in repeated quantitation, for example during serial follow-up or in case of a rest-stress protocol. Therefore, the aim of this study was to assess the scan-rescan reproducibility of 4D flow MRI measurements of kinetic energy, viscous energy loss and vorticity within the LV during diastolic filling in healthy subjects.

\section{Materials and methods}

\section{Study population}

The study protocol was approved by the local Medical Ethical Committee of the Leiden University Medical Center and informed consent was obtained from all participants. Twelve healthy volunteers with no history of cardiac disease were included. All subjects underwent an MRI scan including whole-heart 4D flow MRI between July 2015 and April 2017. The same scanning protocol was performed twice in the same session with 10-min breaks between the scans and repositioning and replanning for every volunteer. Ten of these volunteers were included in a recent study [14]. That study did not report assessment of KE, EL or vorticity.

\section{Cardiovascular magnetic resonance acquisition and data preparation}

Whole-heart 4D flow MRI was obtained on a $3 \mathrm{~T}$ scanner (Ingenia, Philips Medical Systems, The Netherlands) with maximal amplitude of $45 \mathrm{mT} / \mathrm{m}$ for each axis, slew rate of $200 \mathrm{~T} / \mathrm{m} / \mathrm{s}$ and a combination of FlexCoverage Posterior coil in the table top with a dStream Torso coil, providing up to 32 coil elements for signal reception. The orientation of the acquisition of 4D flow data was identical to the 4-chamber orientation (usually double-oblique axial or coronal). Velocity-encoding of $150 \mathrm{~cm} / \mathrm{s}$ in all three directions was used in a standard four-point encoding scheme, spatial resolution $3.0 \times 3.0 \times 3.0 \mathrm{~mm}^{3}$, fieldof-view $400 \mathrm{~mm}$, flip angle $10^{\circ}$, echo time (TE) $3.7 \mathrm{~ms}$, repetition time (TR) $10 \mathrm{~ms}$, true temporal resolution $40 \mathrm{~ms}$, SENSitivity Encoding (SENSE) factor 2 in anterior-posterior direction and Echo Planar Imaging (EPI) readout with a factor 5 . Free breathing was allowed and no respiratory motion compensation was performed. Retrospective gating was used with 30 phases reconstructed to represent one cardiac cycle. Expected scan-time for the 4D flow MRI acquisition for a patient with a heart rate of $60 \mathrm{bpm}$ and 39 slices would be $9 \mathrm{~min}$ and $11 \mathrm{~s}$. This 4D flow MRI sequence with EPI readout has been validated in vivo and in vitro [15] and compared to other 4D flow MRI sequences [16]. Concomitant gradient correction was performed from standard available scanner software. Cine two-dimensional (2D) left 2-chamber, 4-chamber, coronal and sagittal aorta views and a cine multi-2D short-axis stack of slices were acquired, using steady-state free-precession (SSFP) sequences with TE/TR $1.5 / 3.0,350 \mathrm{~mm}$ field-of-view, $45^{\circ}$ flip angle, acquisition resolution $1.9 \times 2.0 \times 8.0 \mathrm{~mm}^{3}$. Retrospective gating was used with 30 phases reconstructed to represent one cardiac cycle. Expected scan-time for the cine multi-2D short-axis acquisition for a patient with a heart rate of $60 \mathrm{bpm}$ and 12 slices would be $1 \mathrm{~min}$. Free breathing was allowed without using motion suppression, three signal averages were taken to minimize effects of breathing motion, which makes the expected scan-time $3 \mathrm{~min}$. Image analysis was performed by one observer (VPK) with over 2 years of experience in MRI and verified by one observer (JJMW) with over 15 years of experience in MRI. The endocardial border was manually traced in all slices and phases in the multislice 2D cine short-axis images and ventricular volume was calculated at the end-diastolic and end-systolic phases using in-house developed MASS software. Papillary muscles were disregarded and assumed to be included in the ventricular volume. $\mathrm{LV}$ in- and outflow was assessed using the 4D flow MRI data with retrospective valve tracking of the mitral and aortic valve, as shown in a recent study 
[14]. Cardiac output (CO) was computed from the 4D flow data as LV outflow $\times$ Heart rate (HR). Beginning and ending of diastolic phases [early diastolic filling (E-filling) and late diastolic filling (A-filling)] were derived from the mitral valve flow-time curves that resulted from retrospective valve tracking. Segmentation of the LV cavity in the 4D flow MRI acquisition, that is required for the energy analysis, was obtained by transforming the available time-varying segmentation of multi-slice cine short-axis acquisition to the 4D flow MRI data. To correct for patient motion related misalignment between the two acquisitions, automated image-based 3D rigid registration was performed using the phase with optimal depiction of the LV cavity in both scans with the Elastix image registration toolbox [17]. Kinetic energy, viscous energy loss and vorticity analysis of segmented LV volumes was done by one investigator (MSME) using in-house developed software.

\section{KE analysis over LV diastole}

The amount of KE during diastolic filling was computed following previously published methods [1]. KE for each voxel within the $\mathrm{LV}$ was computed as $1 / 2 \mathrm{mv}^{2}$, with (m) as the mass representing the voxel volume multiplied by the density of blood $(1.025 \mathrm{~g} / \mathrm{mL})$ and $(\mathrm{v})$ as the 3-directional velocity from 4D flow MRI. For each acquired timephase, volumetric KE was then computed by integrating (by cumulative sum) the computed KE over the segmented 3D $\mathrm{LV}$ volume. In order to quantify KE during diastolic filling, the time-averaged $\mathrm{KE}$ during diastolic phases $\left(\mathrm{KE}_{\mathrm{E} \text {-avg }}\right.$ and $\left.\mathrm{KE}_{\mathrm{A}-\text { avg }}\right)$ and peak $\mathrm{KE}\left(\mathrm{KE}_{\mathrm{E} \text {-peak }}\right.$ and $\left.\mathrm{KE}_{\mathrm{A} \text {-peak }}\right)$, in Joule, were assessed.

\section{Viscous EL analysis over LV diastole}

Following recently published methods [1], we have computed EL from 4D flow MRI using the dissipation terms from the Navier-Stokes energy equations, assuming blood as a Newtonian fluid. The formulae that were used to calculate EL are summarized in."Appendix 1". The time-averaged viscous energy loss rate [EL, in Watt $(\mathrm{W})]$ during E-filling ( $\left.\dot{\mathrm{ELE}}^{-a v g}\right)$

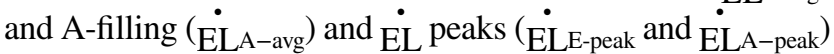
were assessed. We have used the previously reported correlation between $\mathrm{KE}$ and $\dot{\mathrm{EL}}[1]$ as a measure of in-scan consistency. Furthermore, EL over the total diastole ( $\left.\mathrm{EL}_{\text {total diastole }}\right)$ in Joule (J) was computed. Given that the amount of viscous energy lost is proportional to the amount of kinetic energy, we computed a dimensionless energy loss parameter, $\mathrm{EL}_{\text {index }}$, that reflects the amount of viscous energy loss indexed for the average kinetic energy over diastole. $\mathrm{EL}_{\text {index }}$ is a dimensionless index that was used in an earlier echo particle image velocimetry study by Agati et al. [18] to indicate the relative amount of kinetic energy lost to that available over cardiac cycle. In this study, $\mathrm{EL}_{\text {index }}$ was computed over diastole as $\mathrm{EL}_{\text {total diastole }} / \mathrm{KE}_{\text {average diastole, }}$, with $\mathrm{KE}_{\text {average diastole }}$ being the average KE during total diastole. In order to compare our results with a previous study reporting EL values normalized by stroke volume (SV) [1], we also report EL values in the current study as normalized by SV (reported as norm_E

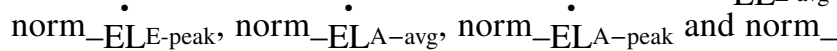
$\left.\mathrm{EL}_{\text {total diastole }}\right)$. To be consistent with the previous study SV was derived from cine SSFP short-axis slices.

\section{Integral vorticity magnitude over LV diastole (vorticity_LV)}

The formulae that were used to calculate the integral vorticity magnitude are shown in "Appendix 1". In short, following previously published work $[8,9]$, for each acquired time-phase, voxel-wise vorticity magnitude $(1 / \mathrm{s})$ was first computed over the segmented LV volume. Then, the instantaneous integral vorticity magnitude was computed as the cumulative sum of voxel-wise vorticity and multiplied by voxel volume to give the integral in (milliliter $\times 1 / \mathrm{sec}$ ond) i.e. $(\mathrm{mL} / \mathrm{s})$. Note that the computed vorticity integral parameter is a scalar quantity and therefore does not take the vorticity direction into account. We will refer to this vorticity integral over the LV as vorticity_LV throughout the text, to differentiate it from voxel-wise vorticity. In order to quantify the integral vorticity_LV over diastolic filling, the time-average and peak vorticity_LV during E-filling (vorticity_LV $V_{\text {E-avg, }}$, vorticity_LV $V_{\text {E-peak }}$, respectively) and A-filling (vorticity_LV $V_{\mathrm{A}-\text { avg }}$, voriticity_LV $\mathrm{A}_{\mathrm{A}-\text { peak }}$, respectively) were computed.

\section{Scan-rescan analysis}

For the scan-rescan analysis, all data was blinded by one observer (VPK) and presented in a random order to the observer (MSME) that performed the energy and vorticity analysis. Scan-rescan analysis was performed to test the reproducibility of (1) KE over E-filling and A-filling; (2) EL over E-filling, A-filling and total diastolic filling and (3) vorticity_LV over E-filling and A-filling.

\section{Statistical analysis}

Data analysis was performed using SPSS Statistics software (v. 23.0 IBM SPSS, Chicago, IL). Variables were tested for normal distribution using the Shapiro-Wilk test. Continuous data was expressed as mean \pm standard deviation (SD) with minimum and maximum values or as median [interquartile range] where suitable. Mean differences were determined for inter-scan comparison and significance was tested by a paired samples $t$ test or, in case of non-normality, the 
Wilcoxon signed-rank test. Differences were computed as: measurement in scan 2-measurement in scan 1 . The coefficient of variation $(\mathrm{CV})$ was calculated with the root mean square method [19]. Correlation between the in-scan and inter-scan measurements done in repeated scans was tested by the Pearson correlation coefficient (r), or the Spearman correlation coefficient $(\rho)$ in case of non-normality of the data. The approach described by Bland and Altman [20] was used to study systematic differences between measurements obtained from the two scans. Agreement between these measurements was assessed by determining the intra-class correlation (ICC) coefficient. Correlation and agreement were classified as follows: $r / \rho$ and ICC $>0.95$ : excellent, 0.95-0.85: strong, 0.85-0.70: good, 0.70-0.5: moderate, $<0.5$ : poor. A $P$ value $<0.05$ was considered statistically significant.

\section{Results}

Volunteer characteristics are shown in Table 1. Heart rate and cardiac output were not significantly different between the two scans (HR: $60.8 \pm 7.8$ vs. $59.9 \pm 6.9$ bpm, $P=0.52$ and $C O$ : $5.5 \pm 0.9$ vs. $5.6 \pm 1.3 \mathrm{~L} / \mathrm{min}, P=0.75)$. $4 \mathrm{D}$ flow MRI data acquisition was successful in all volunteers. Figure $1 \mathrm{a}-\mathrm{c}$ shows cross-sectional mapping of the volumetric measurements of $\mathrm{KE}, \mathrm{EL}$ and vorticity inside the LV at peak E-filling in a standard 4-chambers view. An example of the temporal evolution of $\mathrm{KE}$ and $\dot{\mathrm{EL}}$ over LV diastole is shown in one subject in Fig. 1d.

\section{Intra-scan comparison of energetics (KE vs. EL)}

Figure 2 shows the in-scan comparison of KE versus EL. Comparison of $\mathrm{KE}_{\mathrm{E}-\text { avg }}$ to $\dot{\mathrm{ELE}-a v g}$ showed strong-excellent correlation $(\operatorname{scan} 1: \mathrm{r}=0.92, P<0.001$; scan $2: \rho=0.96$, $P<0.001)$. Also, correlation between $\mathrm{KE}_{\mathrm{A}-\mathrm{avg}}$ and ELA-avg was strong-excellent in both scans (scan 1: $\rho=0.87$, $P<0.001$; scan 2: $\mathrm{r}=0.99, P<0.001)$.

\section{Scan-rescan analysis of kinetic energy and viscous energy loss rate over early diastolic filling}

Detailed results of the scan-rescan tests of $\mathrm{KE}$ and $\dot{\mathrm{EL}}$ assessment over early diastolic filling are shown in Tables 2 and 3 and Fig. 3. Scan-rescan assessment showed poor results for early diastolic filling, as shown in Table 2. Of note, Fig. 3 shows that for $\mathrm{KE}$ and $\dot{\mathrm{EL}}^{-}$assessment over early diastolic filling two subjects showed more distinct differences. Throughout the text we will refer to these two subjects as "Subject 1" and "Subject 2". Detailed scan-rescan information of these two subjects is provided in "Appendix 2". In Fig. 3a, Subject 1 and 2 are indicated as dark $\left(\mathrm{KE}_{\mathrm{E}-\mathrm{avg}}\right)$ and light red triangles $\left(\mathrm{KE}_{\mathrm{E}-\text { peak }}\right)$ and in Fig. 3b, as dark ( $\left.\dot{E}_{\text {EL-avg }}\right)$ and light red triangles ( $\left.\dot{\text { ELE-peak }}\right)$.

Possibly some factors related to the 4D flow MRI acquisition or physiological factors have resulted in these marked differences, therefore we performed an evaluation for a subcohort without Subject 1 and 2. Scan-rescan correlations were much stronger and variation was less for this sub-cohort, as shown in Table $3 . \mathrm{KE}_{\mathrm{E} \text {-avg }}$ and $\dot{\mathrm{ELE}}^{-a v g}$ assessment showed non-significant differences between the two scans, strong $\operatorname{ICCs}\left(\mathrm{KE}_{\mathrm{E}-\mathrm{avg}}: 0.95, P<0.001\right.$ and $\left.\dot{\mathrm{ELE}}_{\mathrm{avg}}: 0.91, P=0.03\right)$ and $\mathrm{CVs} \leq 11 \%\left(\mathrm{KE}_{\mathrm{E}-\mathrm{avg}}: 10 \%\right.$ and $\left.\dot{\mathrm{ELE}}_{\mathrm{E}-\mathrm{avg}}: 11 \%\right)$. norm_ELE-avg showed a strong ICC $(0.90, P=0.001)$ and a CV of $12 \%$. Scan-rescan assessment of $\mathrm{KE}_{\mathrm{E}-\text { peak }}$ and ELE-peak showed nonsignificant differences. The ICC of $\mathrm{KE}_{\mathrm{E}-\text { peak }}$ was good (0.82,

Table 1 Baseline characteristics

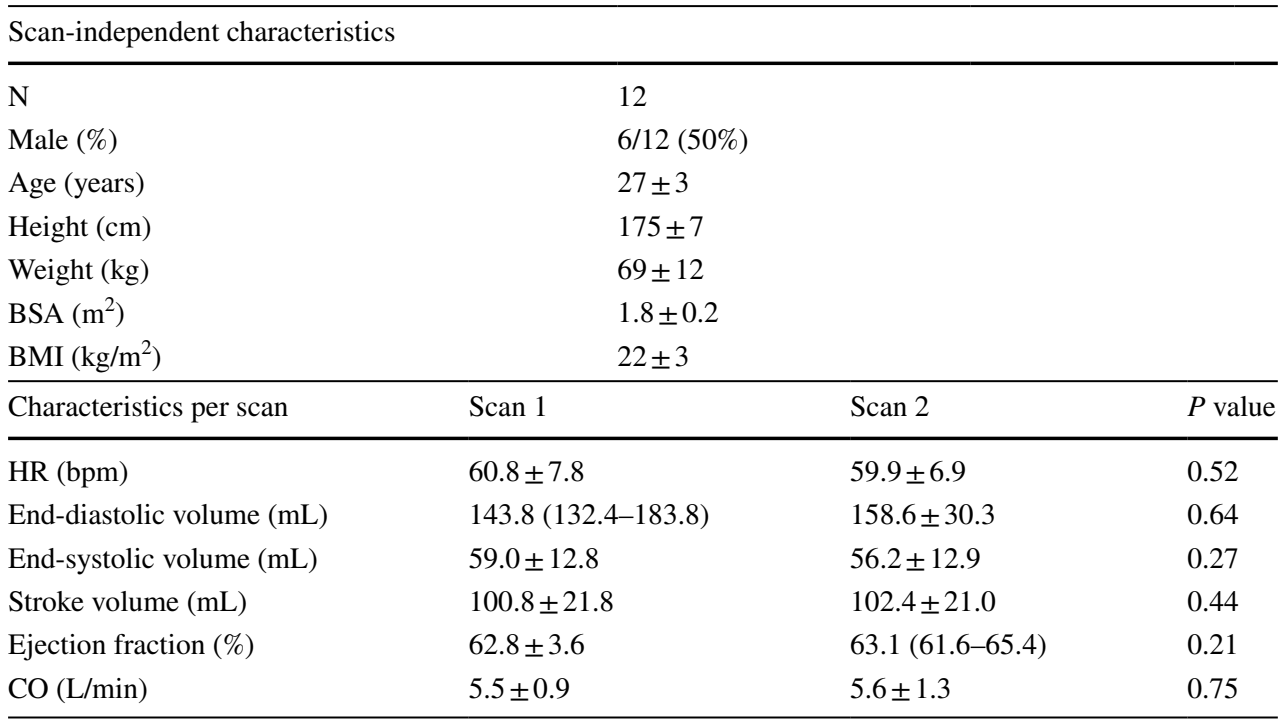

bpm beat per minute, BMI Body Mass Index, BSA body surface area, $C O$ cardiac output, $H R$ heart rate, LV left ventricular 


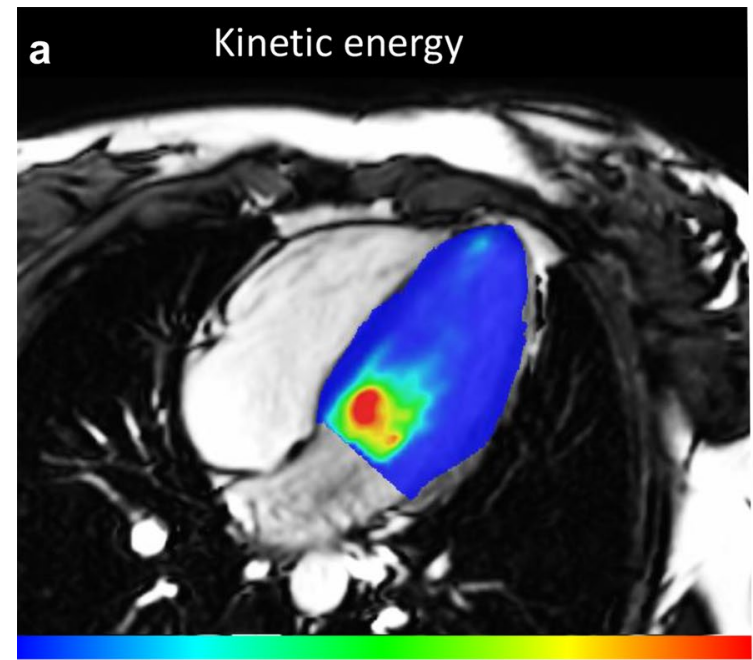

$0 \mu$

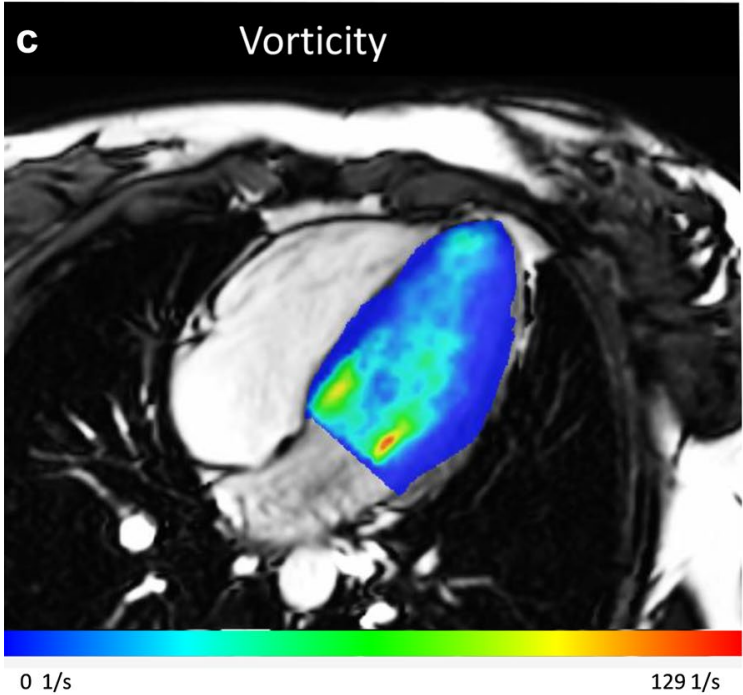

Fig. 1 Maps of left ventricular kinetic energy (KE), viscous energy loss rate (EL) and vorticity over the LV of a healthy female subject (age 20 years) in a standard 4-chambers MRI cross-sectional view, a left ventricular kinetic energy at peak early diastolic filling, b left
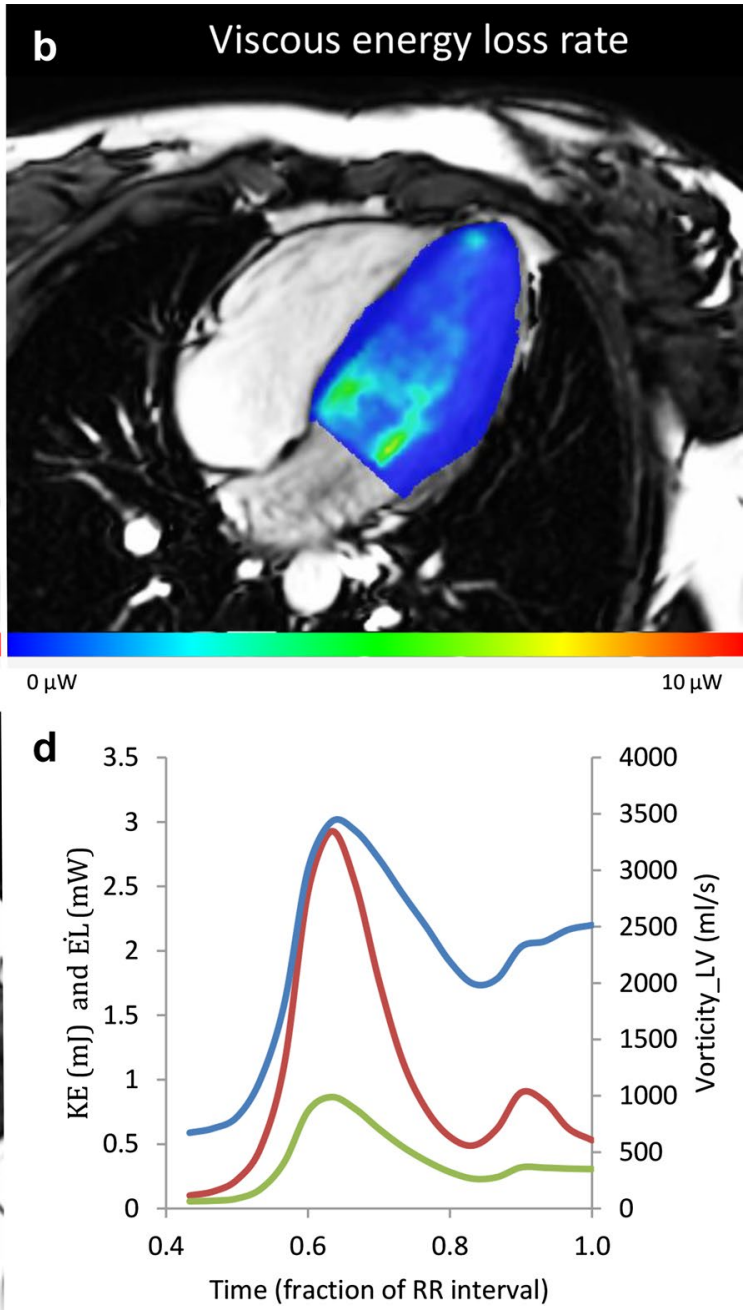

$\mathrm{KE} \rightleftharpoons \mathrm{EL} \longrightarrow$ Vorticity_LV

ventricular viscous energy loss rate at peak early diastolic filling, c left ventricular voxel-wise vorticity at peak early diastolic filling, d temporal evolution of volumetric kinetic energy, viscous energy loss rate and vorticity over LV diastole

now within the same range as the other values [Fig. 3c, indicated as dark $\left(\mathrm{KE}_{\mathrm{A}-\text { avg }}\right)$ or light red triangles $\left.\left(\mathrm{KE}_{\mathrm{A} \text {-peak }}\right)\right]$. The same was observed when assessing $\dot{E L A-a v g}$ and $\dot{E L A-p e a k}$ [Fig. 3d, indicated as dark $(\dot{\mathrm{ELA}-a v g})$ or light red triangles $(\dot{E L A-p e a k})]$. However, to be consistent we repeated the evaluation in the sub-cohort without Subject 1 and Subject 2. Scan-rescan correlations and variation were similar for the sub-cohort without Subject 1 and Subject 2, as shown in Table 3. Reproducibility of $\mathrm{KE}_{\mathrm{A}-\text { avg }}$ and $\dot{\mathrm{ELA}}_{\text {-avg }}$ assessment showed non-significant differences, good ICCs $\left(\mathrm{KE}_{\mathrm{A}-\mathrm{avg}}\right.$ : $0.77, P=0.02$ and $\dot{\text { ELA-avg }}: 0.75, P=0.03)$ and $\mathrm{CVs}$ up to $24 \%\left(\mathrm{KE}_{\mathrm{A}-\mathrm{avg}}: 23 \%\right.$ and $\left.\dot{\mathrm{ELA}}_{-\mathrm{avg}}: 24 \%\right)$. norm_E $\dot{\mathrm{LA}}_{-a v g}$

\section{Scan-rescan analysis of kinetic energy and viscous energy loss rate over late diastolic filling}

Detailed results of the scan-rescan tests of KE and $\dot{\mathrm{EL}}^{\text {assess- }}$ ment over late diastolic filling are shown in Tables 2 and 3 and Fig. 3. Scan-rescan assessment showed good results for late diastolic filling, as shown in Table 2. Subject 1 and 2 are 

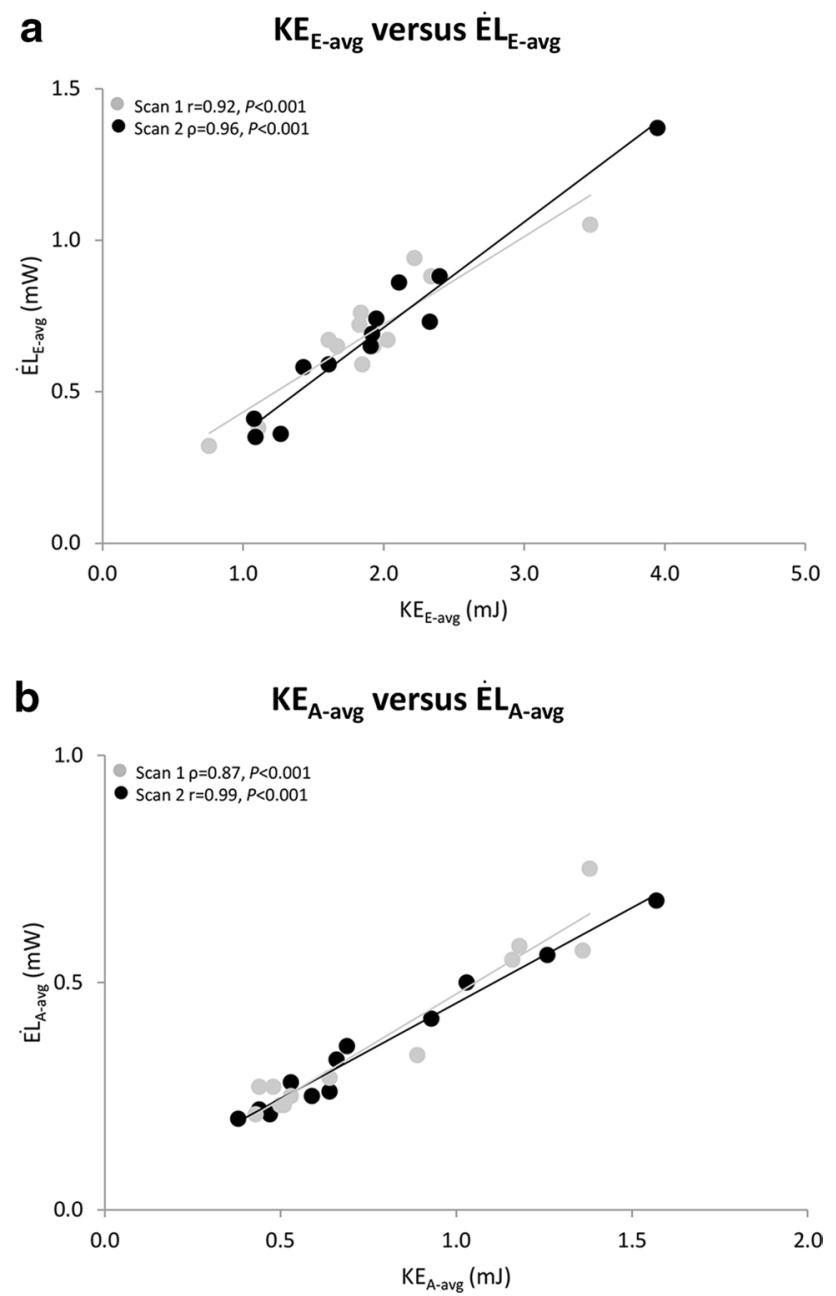

Fig. 2 Scatter plots of kinetic energy (KE) versus viscous energy loss rate (EL) during early diastolic filling and KE versus EL during late diastolic filling. a Scatter plot depicting the correlation between $\mathrm{KE}_{\mathrm{E}-\mathrm{avg}}$ and $\mathrm{EL}_{\mathrm{E}-\mathrm{avg}}$ measured in scan 1 (grey) and scan 2 (black), in all 12 subjects, $\mathbf{b}$ scatter plot depicting the correlation between $\mathrm{KE}_{\mathrm{A}-\mathrm{arg}}$ and $\mathrm{EL}_{\mathrm{A}-\mathrm{avg}}$ measured in scan 1 (grey) and scan 2 (black), in all 12 subjects

showed a non-significant difference between the scans, a good ICC $(0.70, P=0.048)$ and a CV of $22 \%$. Scan-rescan assessment of $\mathrm{KE}_{\mathrm{A}-\text { peak }}$ and $\dot{\mathrm{ELA}-\text { peak }}$ showed non-significant differences, good ICCs $\left(\mathrm{KE}_{\mathrm{A}-\text { peak }}: 0.83, P=0.01\right.$ and ELA-peak: $0.79, P=0.02)$ and $\mathrm{CVs}$ up to $25 \%\left(\mathrm{KE}_{\mathrm{A}-\text { peak }}\right.$ : $23 \%$ and ELA-peak: $25 \%$ ). Lastly, scan-rescan assessment of norm_ELA-peak showed a non-significant difference between the scans, a moderate ICC $(0.64, P=0.08)$ and a CV of $24 \%$.

\section{Scan-rescan analysis of kinetic energy and viscous energy loss over total diastole}

Detailed results of the scan-rescan tests of KE and $\dot{\mathrm{EL}}$ assessment over total diastole $\left(\mathrm{EL}_{\text {total diastole }}\right.$ and
norm_EL $\mathrm{E}_{\text {total diastole }}$ ) and $\mathrm{EL}_{\text {index }}$ are shown in Tables 2 and 3 and Fig. 3. Scan-rescan assessment showed poor results for total diastole, but strong results for $\mathrm{EL}_{\text {index }}$, as shown in Table 2. Scan-rescan correlations were much stronger and variation was less for the sub-cohort without Subject 1 and Subject 2 (Table 3). Scan-rescan assessment of $\mathrm{EL}_{\text {total diastole }}$ showed a non-significant difference between the scans, a strong ICC of $0.91(P=0.001)$ and a CV of $11 \%$. norm $\mathrm{EL}_{\text {total diastole }}$ showed a non-significant difference between the scans, a good ICC $(0.81, P=0.01)$ and a CV of $12 \%$. Figure $3 \mathrm{f}$ shows the Bland-Altman plot of $\mathrm{EL}_{\text {index }}$. For all subjects, scan-rescan assessment of $\mathrm{EL}_{\text {index }}$ showed excellent reproducibility with a small non-significant difference between the scans, a strong ICC of $0.94(P<0.001)$ and a $\mathrm{CV}$ of $9 \%$. When evaluating the sub-cohort without Subject 1 and 2 the results remained similar (ICC: $0.95, P<0.001$ and $\mathrm{CV}: 8 \%$ ).

\section{Scan-rescan analysis of volumetric vorticity_LV over early diastolic filling}

Detailed results of the scan-rescan tests of vorticity_LV assessment over early diastolic filling are shown in Tables 4 and 5 and Fig. 4. Scan-rescan assessment showed moderate results for early diastolic filling as shown in Table 4 . The Bland-Altman plots of the assessment of vorticity_L $\mathrm{LV}_{\mathrm{E} \text {-avg }}$ and vorticity $\mathrm{LV}_{\mathrm{E} \text {-peak }}$ showed higher differences between scan and rescan measurements for Subject 1 and 2 (Fig. 4a, indicated as dark (vorticity_LV $\mathrm{E}_{\mathrm{E}-\mathrm{avg}}$ ) or light red triangles (vorticity_L $\mathrm{LV}_{\mathrm{E} \text {-peak }}$ )).

Scan-rescan correlations were much stronger and variation was less for the sub-cohort without Subject 1 and 2 (Table 5). Scan-rescan assessment of vorticity_LV $\mathrm{V}_{\mathrm{E}-\text { peak }}$ and vorticity $\mathrm{LV}_{\mathrm{E}-\text { avg }}$ showed a non-significant difference between the scans, good-strong ICCs (vorticity_LV $\mathrm{LV}_{\mathrm{E} \text {-peak }}$ : $0.83, P=0.01$ and vorticity_LV $\left.\mathrm{EV}_{\mathrm{E} \text {-avg: }}: 0.95, P<0.001\right)$ and $\mathrm{CVs}$ up to $11 \%$ (vorticity_LV $\mathrm{E}_{\text {E-peak }}: 11 \%$ and vorticity_LV $\mathrm{LV}_{\mathrm{E} \text {-avg }}: 7 \%$ ).

\section{Scan-rescan analysis of volumetric vorticity_LV over late diastolic filling}

Detailed results of the scan-rescan tests of vorticity_LV assessment over late diastolic filling are shown in Tables 4 and 5 and Fig. 4. Scan-rescan assessment showed good results for late diastolic filling as shown in Table 4. Subject 1 and 2 are now within the same range as the other values (Fig. 4b, indicated as dark (vorticity_LV $\mathrm{A}_{\mathrm{A}-\text { avg }}$ ) and light red triangles (vorticity_LV $\mathrm{L}_{\mathrm{A}-\mathrm{peak}}$ )). Scan-rescan variation was similar for the sub-cohort without Subject 1 and 2 (Table 5). Scan-rescan assessment of vorticity_LV $\mathrm{L}_{\mathrm{A}-\text { peak }}$ and vorticity_ $\mathrm{LV}_{\mathrm{A}-\text { avg }}$ showed non-significant differences between the scans, good-strong ICCs (vorticity_LV $\mathrm{LV}_{\mathrm{A}-\text { peak }}: 0.91, P=0.001$ and vorticity_LV $\left.\mathrm{A}_{\mathrm{A}-\mathrm{avg}}: 0.89, P=0.002\right)$ and $\mathrm{CVs}$ of $11 \%$. 


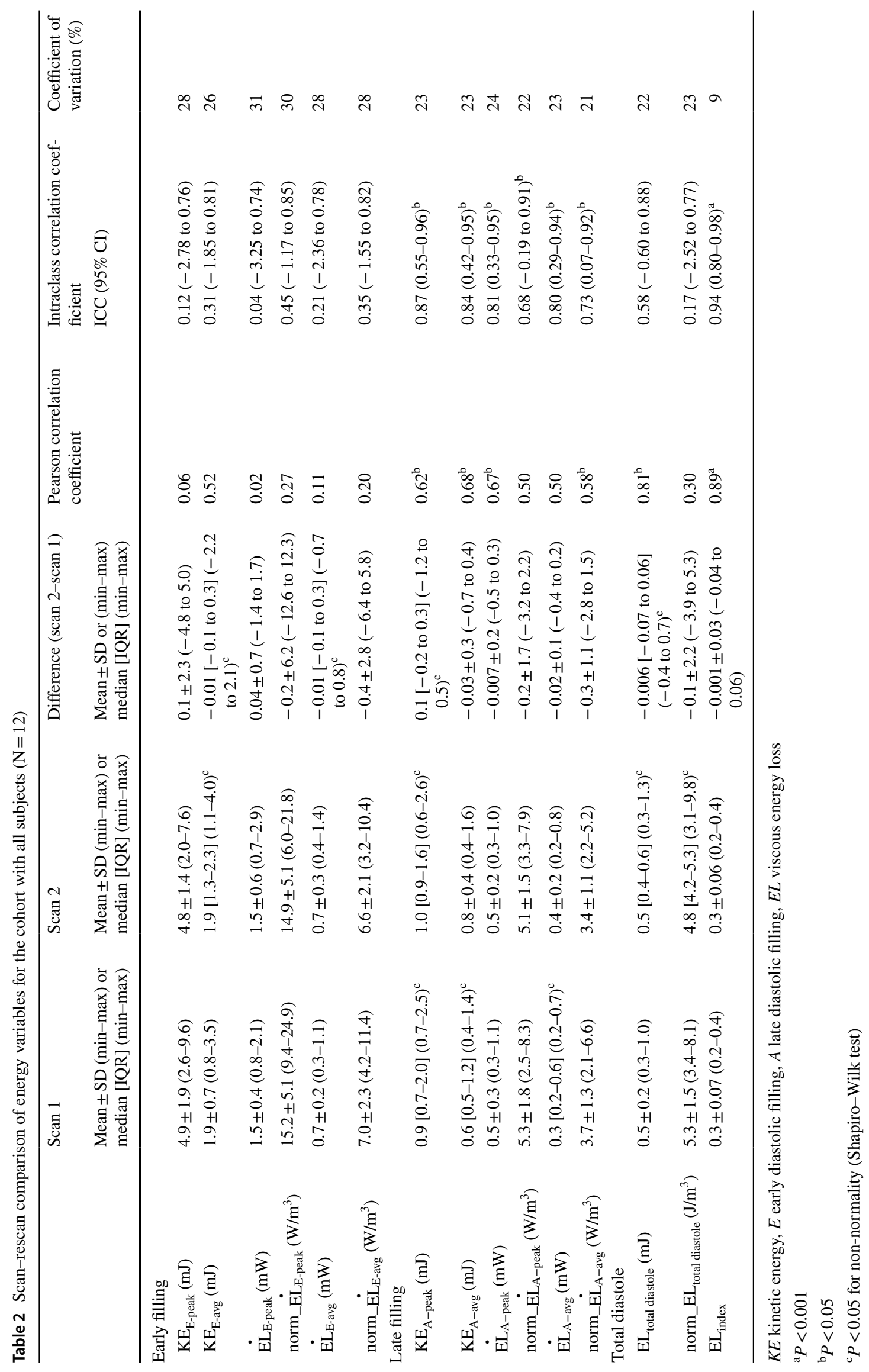




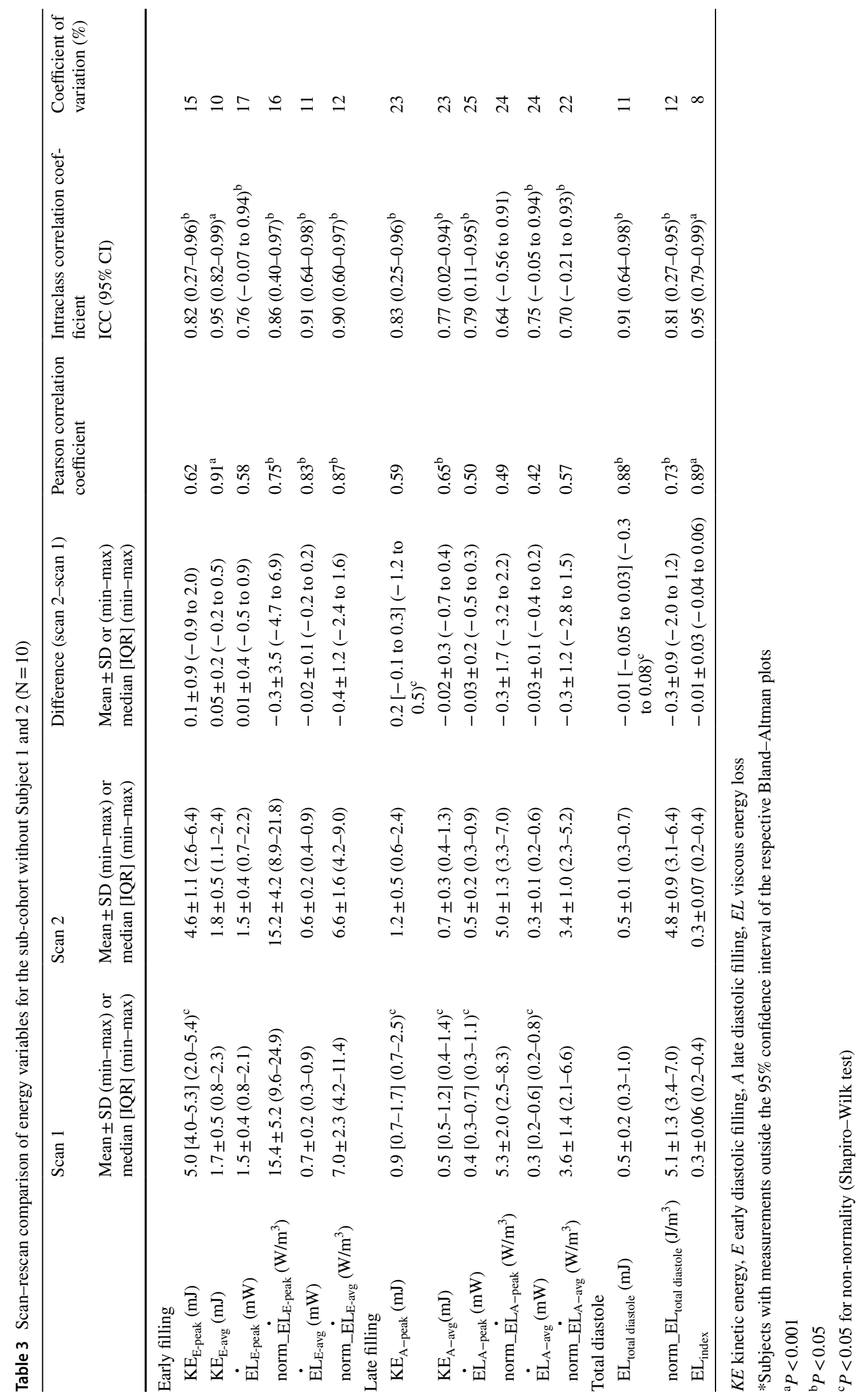




\section{Discussion}

In the current study, kinetic energy, viscous energy loss and vorticity inside the left ventricle during diastole are derived from 4D flow MRI and scan-rescan reproducibility of these parameters is tested. Scan-rescan reproducibility is essential for the clinical application of a parameter since it reflects the reliability of a measurement and feasibility of repeated measurement evaluations. The main findings of this study are: (1) internal consistency between kinetic energy and viscous energy loss is strong-excellent in both scans during early and late diastolic filling; (2) In the majority (10 out of 12) of subjects, reproducibility of peak and average kinetic energy and viscous energy loss during early, late and total diastolic filling shows non-significant differences but with a good to excellent agreement (by means of ICC) and CVs up to $25 \%$; (3) In the studies parameters, time-averaged measurements over E- and A-filling show stronger reproducibility than peak measurements. (4) For all subjects, $E_{\text {index }}$ shows good reproducibility with a small non-significant difference between the scans, strong agreement and a CV of 9\%; (5) Assessment of volumetric vorticity over the left ventricle during early and late diastolic filling shows non-significant differences, good-excellent ICCs and CVs up to $11 \%$.

In the Bland-Altman plots for E-filling parameters, measurements obtained in two subjects (Subject 1 and 2 in Appendix 2) showed distinct higher differences between scan and rescan measurements than the measurements of the majority of the cohort. These high differences had an impact on the scan-rescan reproducibility for $\mathrm{KE}$ and $\dot{\mathrm{EL}}$ measures. This impact seemed to be largest during early diastolic filling, where reproducibility was much higher for the sub-cohort without Subject 1 and 2. However, during late diastolic filling, reproducibility was similar. There is no obvious explanation for the measurements that showed distinct higher differences between scan and rescan measurement, but this could be related to the technical restrictions of the 4D flow MRI acquisition or to physiological differences between scans, or a combination of both. It is to be expected that physiological differences were small as all subjects are healthy volunteers that were scanned twice under the same circumstances with only a short break between the scans $( \pm 10 \mathrm{~min})$ and heart rate was not significantly different between both scans. Still, subtle physiological differences could result in poor scan-rescan reproducibility for these few cases. Table 6 in "Appendix 2" shows the HR, LV outflow and $\mathrm{CO}$ of all subjects. Subject 1 and 2 present the highest $\mathrm{CO}$ differences, however there are other subjects with $\mathrm{CO}$ differences within the same range. Table 7 in "Appendix 2" shows subjectspecific scan-rescan information from the mitral valve flow curves. Subject 1 and 2 have the highest difference in area under the curve of the E-filling and peak filling rate of the E-filling. This could indicate that differences may be related to technical restrictions of the acquisition, as the E-filling duration, E peak filling rate and the area-under-the-curve are sensitive to temporal and spatial resolution. These observations are important to take into consideration when evaluating flow energetics and vorticity in a research or clinical setting.

\section{Kinetic energy (KE) over LV diastole}

Multiple 4D flow MRI studies showed that patients with LV dysfunction present altered flow patterns through the LV with impaired preservation of inflow KE to the end of diastole and altered KE-time curves (the amount of KE inside the LV during each time step over the total cardiac cycle) $[4,6,7$, 21], even in patients with normal to mild LV remodeling and normal to mildly depressed LV systolic function [7]. These $\mathrm{KE}$ changes in the LV could be a valuable diagnostic marker to evaluate diastolic function and might be useful for early detection of deteriorating ventricular function $[1,4-7,21$, 22], which could reduce patient morbidity and mortality [23]. However, there is a lack of studies validating LV KE derived from 4D flow MRI in a scan-rescan setting. Therefore, reliability and reproducibility of KE measurements from 4D flow MRI in a repeated scan setting remains largely unknown. Kanski et al. [24] compared mean KE and peak KE between two scans (with and without respiratory gating) with the aim to evaluate the impact of respiratory gating on KE measurements. They found a strong correlation between the KE measurements in both scans. The current study differs from the study by Kanski et al. [24] in that we used the same protocol for both scans. In the current study, moderate-strong correlation was found between KE measurements in both scans. Absolute values of $\mathrm{KE}_{\mathrm{E} \text {-peak }}$ and $\mathrm{KE}_{\mathrm{A}-\text { peak }}$ reported in the current study are in agreement with previous studies [1, 25, 26].

In the current study we showed the reproducibility of the KE measurements assessed with 4D flow MRI with good-strong agreement (by means of ICCs), however substantial CVs up to $23 \%$ were also found. Based on the Bland-Altman analysis the variability of $\mathrm{KE}_{\mathrm{E} \text {-avg }}$ and $\mathrm{KE}_{\mathrm{A} \text {-avg }}$ was less than the variability of $\mathrm{KE}_{\mathrm{E}-\text { peak }}$ and $\mathrm{KE}_{\mathrm{A}-\text { peak }}$ as shown by the smaller limits of agreement. This might be expected, given that the definition of average is computed over multiple time points and therefore evens out the variations more than a single-time measure such as the peak, especially when the definition of the peak also is affected by the temporal resolution of the 4D flow MRI data.

\section{Viscous EL over LV diastole}

The assessment of intracardiac EL could provide crucial details on the function of the heart apart from the standard MRI parameters and could be used to further unravel the influence of complex surgery for congenital heart defects [1].

To our knowledge, this is the first study assessing scan-rescan reproducibility of in vivo LV EL over diastole 

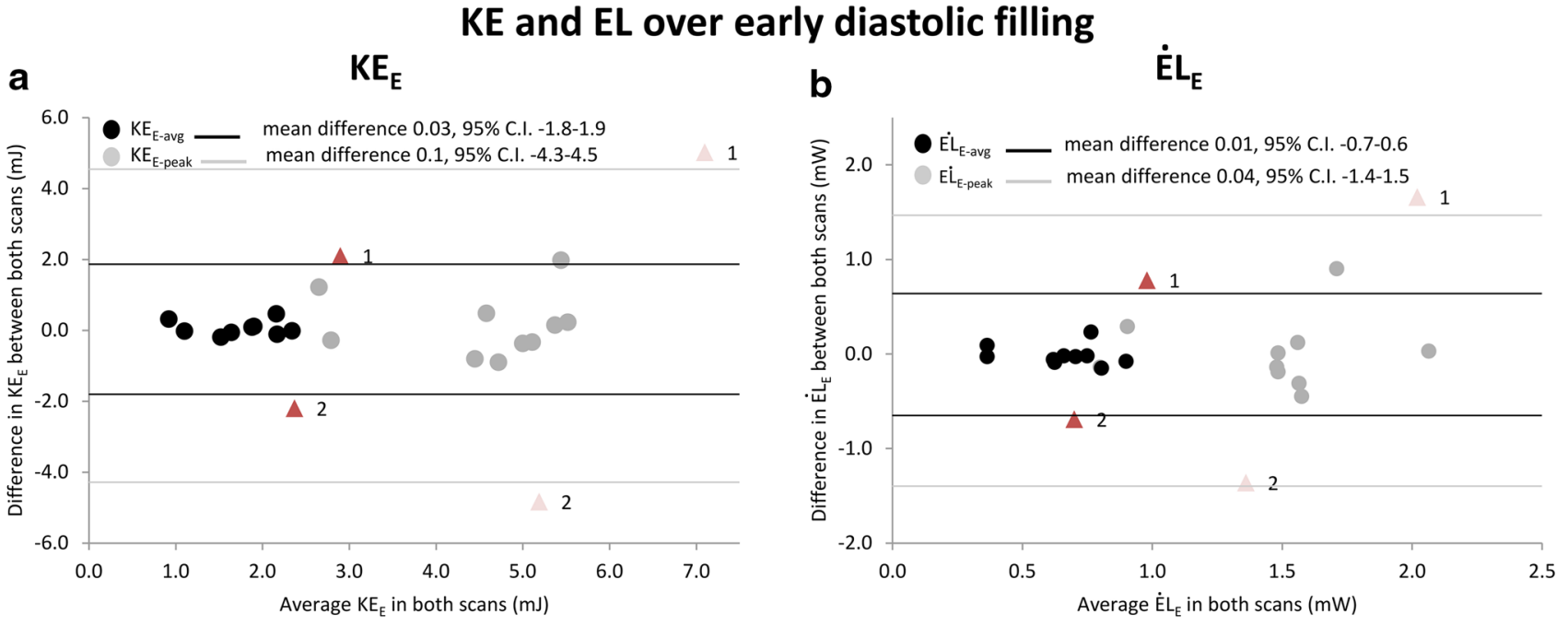

KE and EL over late diastolic filling
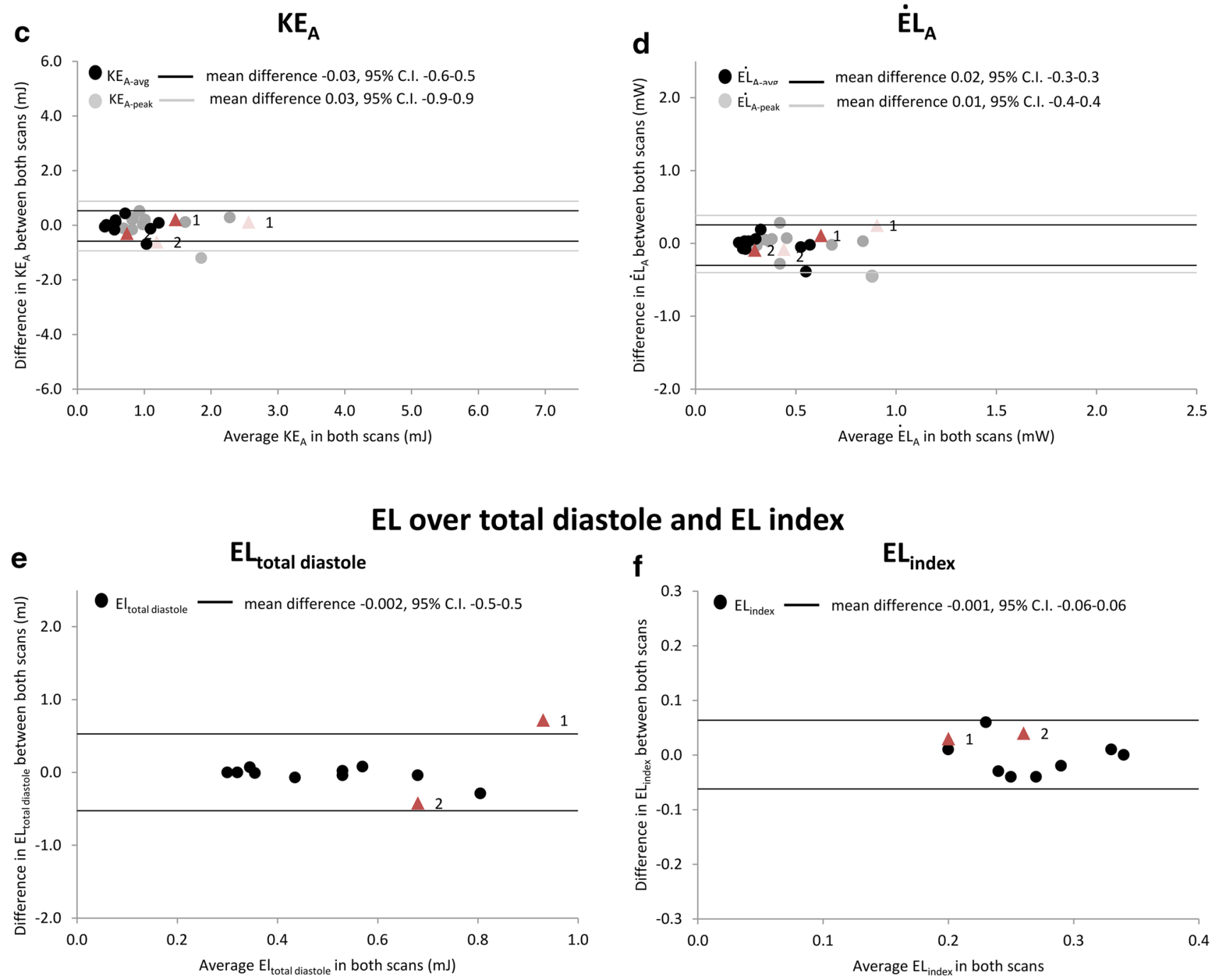
4Fig. 3 Bland-Altman plots of kinetic energy (KE) and viscous energy loss rate $(\mathrm{EL})$ at peak early diastolic filling ( $\mathrm{KE}_{\mathrm{E}-\text { peak }}$ and $\left.\dot{\mathrm{EL}}_{\mathrm{E} \text {-peak }}\right)$ and peak late diastolic filling $\left(\mathrm{KE}_{\mathrm{A}-\text { peak }}\right.$ and $\left.\mathrm{EL}_{\mathrm{A}-\text { peak }}\right), \mathrm{EL}_{\text {total diastole }}$ and $\mathrm{EL}_{\text {index }}$. a Bland-Altman plot depicting the agreement between $\mathrm{KE}_{\mathrm{E} \text {-avg }}$ (black) and $\mathrm{KE}_{\mathrm{E}-\text { peak }}$ (grey) in scan 1 and scan 2 . The subjects with distinct higher differences between scan and rescan measurements (Subject 1 and 2) are depicted as red triangles $\left(\mathrm{KE}_{\mathrm{E} \text {-avg }}\right.$ dark red; $\mathrm{KE}_{\mathrm{E}-\text { peak }}$ light red). b Bland-Altman plot depicting the agreement between ELE-avg (black) and ELE-peak (grey) in scan 1 and scan 2. Subject 1 and 2 are depicted as red triangles $\left(\mathrm{KE}_{\mathrm{E} \text {-avg }}\right.$ dark red; $\mathrm{KE}_{\mathrm{E} \text {-peak }}$ light red). c Bland-Altman plot depicting the agreement between $\mathrm{KE}_{\mathrm{A}-\mathrm{avg}}$ (black) and $\mathrm{KE}_{\mathrm{A}-\text { peak }}$ (grey) in scan 1 and scan 2. Subject 1 and 2 are depicted as red triangles $\left(\mathrm{KE}_{\mathrm{A} \text {-avg }}\right.$ dark red; $\mathrm{KE}_{\mathrm{A} \text {-peak }}$ light red). d Bland-Altman plot depicting the agreement between $\dot{E L}_{\mathrm{A}-\text { avg }}$ (black) and ELA-peak (grey) in scan 1 and scan 2. Subject 1 and 2 are depicted as red triangles (EL $\dot{E L}_{\mathrm{A}-a v g}$ dark red; $\dot{\mathrm{EL}}_{\mathrm{A}-\mathrm{peak}}$ light red). e Bland-Altman plot depicting the agreement between $\mathrm{EL}_{\text {total diastole }}$ in scan 1 and scan 2. Subject 1 and 2 are depicted as red triangles. $\mathbf{f}$ Bland-Altman plot depicting the agreement between $\mathrm{EL}_{\text {index }}$ in scan 1 and scan 2. Subject 1 and 2 are depicted as red triangles

assessed by 4D flow MRI. Results of norm_ELE-peak in the current study correspond well with that of a different healthy controls cohort of a recent 4D flow MRI study by Elbaz et al. [1] Also norm_ELE-avg values are consistent. Furthermore, both results of norm_ELA-peak, as well as norm_EL_-Evg are similar to previously reported numbers [1].

Both $\mathrm{EL}_{\text {total diastole }}$ and norm_EL total diastole are slightly higher in our study than in the study by Elbaz et al. [1]. However, results remain in the same range. These differences in results could be explained in part by differences in heart rate between the volunteers of this study and those of the previous study. This is because the total viscous energy loss over diastole is computed over the time period between the first and the last phase of the diastole (Eq. 3) in "Appendix 1") and heart rate mainly affects the duration of diastole. Still, the EL-time curve over total diastole in the current study (Fig. 1d) agrees well with reported in vivo [1] and in vitro [2] EL time curves.

In this study we demonstrated the reproducibility of the EL parameters with moderate-strong ICCs and substantial CVs of up to $25 \%$. Similar to KE, the Bland-Altman analysis shows that the variability of $\dot{E L E-a v g}$ and $\dot{E}$ EA-avg was less than the variability of ELE-peak and ELA-peak

The amount of energy lost over diastole relative to the average kinetic energy as measured by means of $\mathrm{EL}_{\text {index, }}$, shows good reproducibility for all subjects with an ICC of 0.94 $(P<0.001)$ and an $\mathrm{CV}$ of $9 \%$. The Bland-Altman plot shows that all subjects are within the same range of differences. This suggests that among tested parameters $\mathrm{EL}_{\text {index }}$ is the least sensitive to subtle physiological variations or discrepancies affected by technical limitations of the 4D flow MRI data in the healthy subjects, which might have affected the lesser reproducibility of KE and EL in some subjects. This observation could be attributed to the fact that $\mathrm{EL}_{\text {index }}$ is a dimensionless parameter concerned with the relative changes in EL to KE and not with their absolute changes as in other tested parameters. As such, the reported reproducibility of $\mathrm{EL}_{\text {index }}$ could also be considered as another reflection of a good internal consistency in this study. Although a similar $\mathrm{EL}_{\text {index }}$ parameter was reported in a previous study and was shown to be significantly altered in patients with acute myocardial infarction [18], $\mathrm{EL}_{\text {index }}$ was computed in that study from $2 \mathrm{D}$ echo particle image velocimetry and over the complete cardiac cycle as compared to this study's volumetric measurement from 4D flow MRI and over diastole only. Therefore, it is not possible to perform a direct comparison with the published results of that study.

\section{Vorticity inside the LV during diastolic filling}

LV vortex quantification parameters, such as vorticity, could be useful in the assessment of LV and RV diastolic (dys-)function [1, 8, 9, 11, 12]. In recent studies, vorticity was shown to be a marker of diastolic dysfunction, both in the LV [9] and the RV [8] of patients with pulmonary hypertension.

To our knowledge, no previous study is available on assessing scan-rescan reproducibility of in vivo vorticity_LV over diastole from 4D flow MRI. Fenster et al. [8] assessed vorticity inside the RV using the integral of vorticity magnitude over the volumes and found results in the same order of magnitude as the results in the current study. In a recent paper by Schafer et al. [9] LV vorticity in healthy subjects was assessed. However, it is not clear whether this was computed over the whole LV volume and therefore we cannot compare results to our measurements. The current study showed scan-rescan reproducibility of integral vorticity_LV during E-filling as well as during A-filling with good-excellent ICCs and CVs up to $11 \%$. Based on the Bland-Altman analyses, similar to the $\mathrm{KE}$ and EL results, the variability of the average of the vorticity_L $\mathrm{LV}_{\mathrm{E} \text {-avg }}$ and vorticity_LV $\mathrm{A}_{\mathrm{A}-\mathrm{avg}}$ was less than the variability of the peaks vorticity_LV $V_{\text {E-peak }}$ and vorticity_LV $\mathrm{A}_{\mathrm{A} \text {-peak }}$. Vorticity_LV $\mathrm{E}_{\mathrm{E} \text {-avg }}$ shows less variability than vorticity_LV $\mathrm{A}_{\mathrm{A}-\mathrm{avg}}$ but vorticity_LV $\mathrm{E}_{\text {E-peak }}$ shows slightly more variability than vorticity_LV $\mathrm{LV}_{\mathrm{A}-\text { peak}}$.

\section{Technical considerations}

The post-processing steps that are followed for obtaining these flow energetics and vorticity parameters involve manual segmentation, registration and valve tracking. Manual segmentation on cine short axis images can be performed with excellent reproducibility [27]. In this study, manual segmentation was performed by one observer with over 2 years of experience in MRI and verified by another observer with over 15 years of experience, with validated software [28], which warrants high accuracy. Next, to correct for patient motion related misalignment and minimize errors between the cine short axis and the 4D flow acquisitions, 


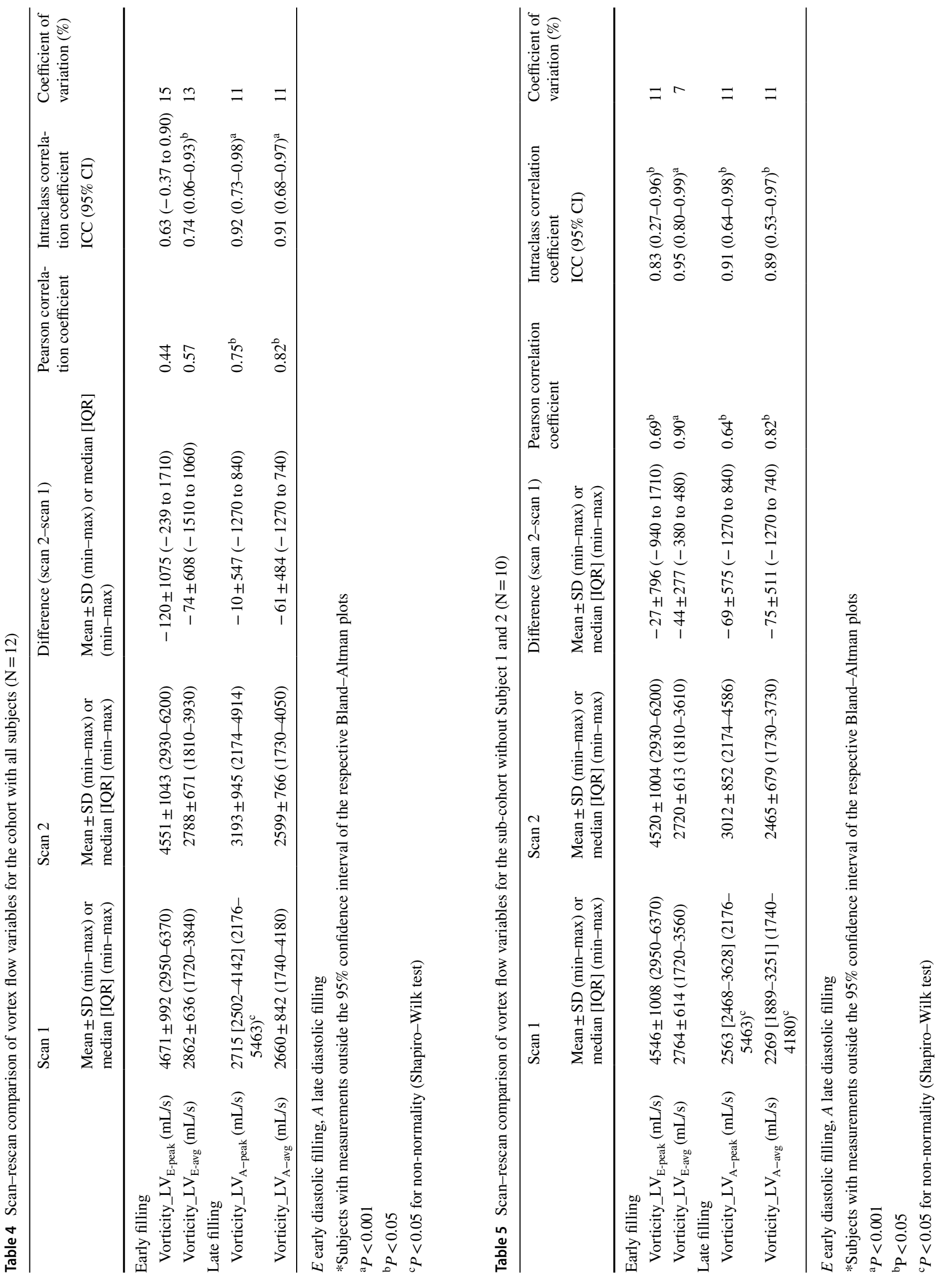



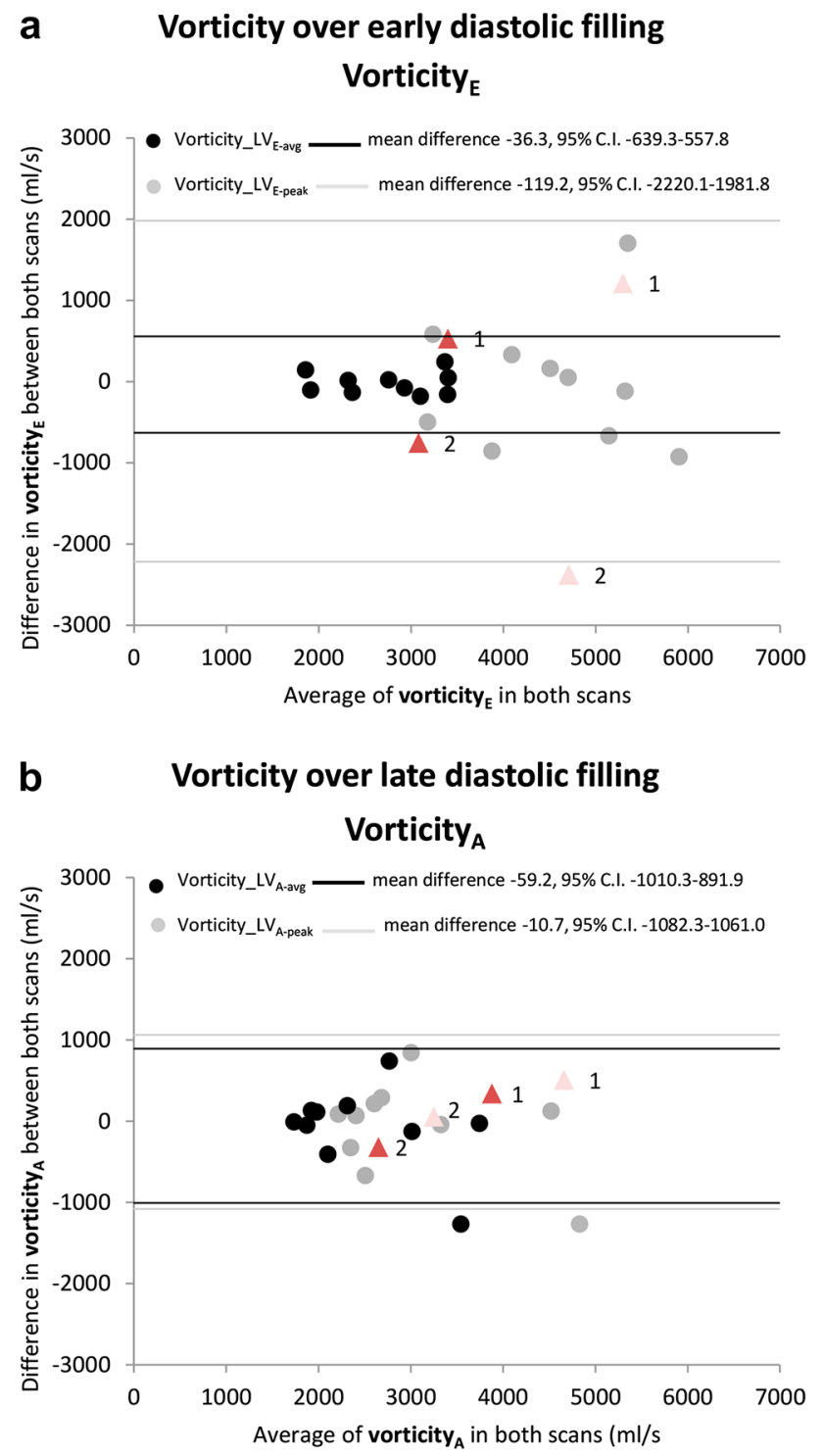

Fig. 4 Bland-Altman plots of volumetric vorticity over peak early filling (vorticity_LV $V_{\mathrm{E}-\text { avg }}$ and vorticity_LV $\mathrm{LV}_{\mathrm{E} \text {-peak }}$ ) and late filling (vorticity_LV $\mathrm{LV}_{\mathrm{A}-\text { avg }}$ and vorticity_LV $\mathrm{LV}_{\mathrm{A}-\text { peak }}$ ). a Bland-Altman plot depicting the agreement between vorticity_LV $\mathrm{E}_{\mathrm{E}-\mathrm{avg}}$ (black) and vorticity_LV $L V_{\text {E-peak }}$ (grey) in scan 1 and scan 2. Subject 1 and 2 are depicted as red triangles (vorticity_LV $\mathrm{LV}_{\mathrm{E}-\mathrm{avg}}$ dark red; vorticity_ $\mathrm{LV}_{\text {E-peak }}$ light red). b Bland-Altman plot depicting the agreement vorticity_LV $V_{\mathrm{A}-\mathrm{avg}}$ (black) and vorticity_LV $\mathrm{A}_{\mathrm{A}-\text { peak }}$ (grey) in scan 1 and scan 2. Subject 1 and 2 are depicted as red triangles (vorticity_ $\mathrm{LV}_{\mathrm{A}-\text { avg }}$ dark red; vorticity_LV $\mathrm{LV}_{\mathrm{A}-\text { peak }}$ light red)

automated image-based 3D rigid registration was performed using the validated Elastix image registration toolbox [17]. Another post-processing step requires retrospective valve tracking and mitral flow velocity mapping for assessing the beginning and ending of diastole. The reproducibility and observer variability of this semi-automated method was shown to be excellent $[14,15]$.

\section{Limitations}

A limitation of this study is the small number of subjects. Furthermore, no patients were scanned in this study as this is difficult to assess in clinical research. In most of our patient studies, 4D flow MRI is part of a clinical CMR evaluation of about 75-90 min, sometimes involving the use of contrast for late gadolinium enhancement and sometimes involving a Dobutamine rest/stress protocol. Scan-rescan evaluation in such cases would imply repeating some of these evaluations as well. Furthermore, repeating 4D flow MRI for scan-rescan purposes would imply an additional scan-time of approximately 30 min (because of replanning), which makes the total scan-time too long to keep the patient in the same physiological state. Another limitation is that the influence of a different scanning protocol or scanner was not assessed. However, it is important to note that in the current study the aim was to assess scan-rescan reproducibility by using the exact same protocol and the same scanner machine twice. The use of different scanners or scanning protocols could result in altered reproducibility, which should be evaluated in future studies.

In conclusion, left ventricular kinetic energy and viscous energy loss quantification from 4D flow MRI in healthy volunteers shows strong-excellent in-scan consistency. Scan-rescan assessment of left ventricular kinetic energy, viscous energy loss and vorticity shows overall good agreement in the majority of the scanned subjects. Nevertheless, in two out of twelve subjects, considerable variation between the scans was found. Agreement of A-filling measurements is better than E-filling between scans in the studied parameters. Furthermore, time-averaged measurements over early and late filling show better reproducibility compared to peak measurements. Strong reproducibility for all cases is found in the dimensionless index, $\mathrm{EL}_{\text {index }}$, that measures the ratio of the amount of viscous energy lost relative to the average kinetic energy over diastole. $\mathrm{EL}_{\text {index }}$ seems to be less influenced by technical and/ or slight physiological differences between scans and may therefore be a useful parameter of energetics for future studies.

Acknowledgements The authors thank professor Dr. S. le Cessie for statistical advice.

Funding V.P. Kamphuis and R.L.F. van der Palen are financially supported by grants from the Dutch Heart Foundation (Grant Numbers 2013T091 and 2014T087, respectively). J.J.M. Westenberg is financially supported by a Grant of ZonMw (Project Number 104003001).

\section{Compliance with ethical standards}

Conflict of interest The authors declare that they have no conflict of interest.

Open Access This article is distributed under the terms of the Creative Commons Attribution 4.0 International License (http://creativecommons.org/licenses/by/4.0/), which permits unrestricted use, 
distribution, and reproduction in any medium, provided you give appropriate credit to the original author(s) and the source, provide a link to the Creative Commons license, and indicate if changes were made.

\section{Appendix 1}

\section{Viscous energy loss computation from 4D flow MRI}

Given the acquired velocity field $v$, the rate of viscous energy loss $(\dot{E L})$ in watt $(W)$ and the total energy loss $\left(\mathrm{EL}_{\text {total }}\right)$ in joule (J) over a given period of time $T$ can be computed from $4 \mathrm{D}$ flow MRI using the viscous dissipation function $\Phi_{v}$ in the Newtonian Navier-Stokes energy equations:

$$
\begin{aligned}
\Phi_{v}= & \frac{1}{2} \sum_{i=1}^{3} \sum_{j=1}^{3}\left[\left(\frac{\partial v_{i}}{\partial x_{j}}+\frac{\partial v_{j}}{\partial x_{i}}\right)-\frac{2}{3}(\nabla \cdot v) \delta_{i j}\right]^{2}, \\
& \left\{\begin{array}{l}
\delta_{i j}=1, \text { if } i=j \\
\delta_{i j}=0, \text { if } i \neq j
\end{array}\left[s^{-2}\right]\right.
\end{aligned}
$$

$\Phi_{v}$ represents the rate of viscous energy dissipation per unit volume. $i, j$ correspond to the principal velocity directions $\mathrm{x}, \mathrm{y}, \mathrm{z} . \nabla \cdot v$ denotes the divergence of the velocity field. Therefore, the rate of viscous energy loss ( $\dot{\mathrm{EL}})$ in Watt at an acquired time phase $\mathrm{t}$ can be computed as:

$$
\dot{\mathrm{EL}_{\mathrm{t}}}=\mu \sum_{i=1}^{M} \Phi_{v} \mathrm{~L}_{\mathrm{i}}[\operatorname{Watt}(\mathrm{W})]
$$

assuming the blood as a Newtonian fluid, the dynamic viscosity is $\mu=0.004 \mathrm{~Pa} \mathrm{~s}, \mathrm{~N}$ as the total number of voxels in the given domain of interest (e.g. LV), $\mathrm{L}_{\mathrm{i}}$ as the voxel volume.
As a result, the total viscous energy loss (EL) in joules over time period $\mathrm{T}$ starting at phase $\mathrm{t}_{\text {start }}$ and ending at $\mathrm{t}_{\text {end }}$ can be computed as:

$\mathrm{EL}_{\mathrm{T}}=\sum_{\mathrm{d}=\mathrm{t}_{\text {start }}}^{\mathrm{t}_{\text {end }}} \dot{\mathrm{EL}_{\mathrm{d}}} \mathrm{p}_{\mathrm{d}}[\operatorname{Joule}(\mathrm{J})]$

with $\mathrm{p}_{\mathrm{d}}$ the time step (temporal resolution) of the acquired 4D flow MRI.

\section{Vorticity computation from 4D flow MRI}

If $u, v, w$ denote the three velocity field components acquired from 4D flow MRI over the principal velocity directions $x, y, z$, respectively, the vorticity $\left(\omega_{i, t}\right)$ at voxel $i$ of an acquired time phase $t$

$\omega_{i, t}=\left(\frac{\partial w_{i, t}}{\partial y_{i, t}}-\frac{\partial v_{i, t}}{\partial z_{i, t}}, \frac{\partial u_{i, t}}{\partial z_{i, t}}-\frac{\partial w_{i, t}}{\partial x_{i, t}}, \frac{\partial v_{i, t}}{\partial x_{i, t}}-\frac{\partial u_{i, t}}{\partial y_{i, t}}\right)[1 / \mathrm{s}]$

Then, Vorticity_LV denoting the integral sum of vorticity over the segmented LV volume at an acquired time phase $t$ in liter $(\mathrm{mL})$ per second (s) can be computed as

Vorticity_LV $\mathrm{L}_{\mathrm{t}}=\sum_{i=1}^{M}\left|\omega_{i, t}\right| L_{i, t}[\mathrm{~mL} / \mathrm{s}]$

With $\left|\omega_{i, t}\right|$ as the magnitude of the vorticity vector, $M$ as the total number of voxels in the segmented LV volume and $L_{i, t}$ as the voxel volume.

\section{Appendix 2}

\begin{tabular}{|c|c|c|c|c|c|c|c|c|c|}
\hline Subject & HR 1 (bpm) & $\begin{array}{l}\text { HR 2 } \\
\text { (bpm) }\end{array}$ & $\begin{array}{l}\text { Difference } \\
\text { HR (bpm) }\end{array}$ & $\begin{array}{l}\text { LV outflow } \\
1(\mathrm{~mL})\end{array}$ & $\begin{array}{l}\text { LV outflow } \\
2(\mathrm{~mL})\end{array}$ & $\begin{array}{l}\text { Difference LV } \\
\text { outflow (mL) }\end{array}$ & $\mathrm{CO} 1(\mathrm{~L} / \mathrm{min})$ & $\mathrm{CO} 2(\mathrm{~L} / \mathrm{min})$ & $\begin{array}{l}\text { Difference } \\
\text { CO }(\%)\end{array}$ \\
\hline $1^{\mathrm{a}}$ & 67.1 & 69.6 & 2.5 & 104.1 & 124.8 & 20.7 & 7.0 & 8.7 & 24.4 \\
\hline $2^{\mathrm{a}}$ & 53.8 & 51.6 & -2.2 & 112.9 & 94.5 & -18.4 & 6.1 & 4.9 & -19.7 \\
\hline 3 & 58.0 & 51.8 & -6.2 & 110.0 & 126.4 & 16.4 & 6.4 & 6.6 & 2.7 \\
\hline 4 & 61.5 & 59.1 & -2.4 & 81.5 & 72.4 & -9.1 & 5.0 & 4.3 & -14.7 \\
\hline 5 & 71.9 & 66.2 & -5.7 & 68.4 & 66.0 & -2.4 & 4.9 & 4.4 & -11.2 \\
\hline 6 & 50.5 & 52.9 & 2.4 & 100.7 & 108.6 & 7.9 & 5.1 & 5.7 & 12.9 \\
\hline 7 & 63.7 & 60.4 & -3.3 & 104.0 & 107.8 & 3.8 & 6.6 & 6.5 & -1.7 \\
\hline 8 & 57.7 & 53.1 & -4.6 & 85.4 & 86.4 & 1.0 & 4.9 & 4.6 & -6.9 \\
\hline 9 & 71.1 & 72.1 & 1.0 & 75.3 & 84.4 & 9.1 & 5.4 & 6.1 & 13.7 \\
\hline 10 & 68.0 & 61.3 & -6.7 & 85.0 & 81.8 & -3.2 & 5.8 & 5.0 & -13.3 \\
\hline 11 & 48.5 & 58.3 & 9.8 & 90.3 & 88.7 & -1.5 & 4.4 & 5.2 & 18.2 \\
\hline 12 & 58.0 & 62.0 & 4.0 & 71.1 & 76.3 & 5.2 & 4.1 & 4.7 & 14.7 \\
\hline
\end{tabular}

See Tables 6 and 7.

Table 6 Subject specific scan-rescan assessment

Differences were calculated as: value scan 2 - value scan 1

$A$ late diastolic filling, $A U C$ area under the cure, $C O$ cardiac output, $E$ early diastolic filling, $H R$ heart rate

${ }^{\text {a }}$ Subjects with marked high differences for the assessment of early filling parameters 


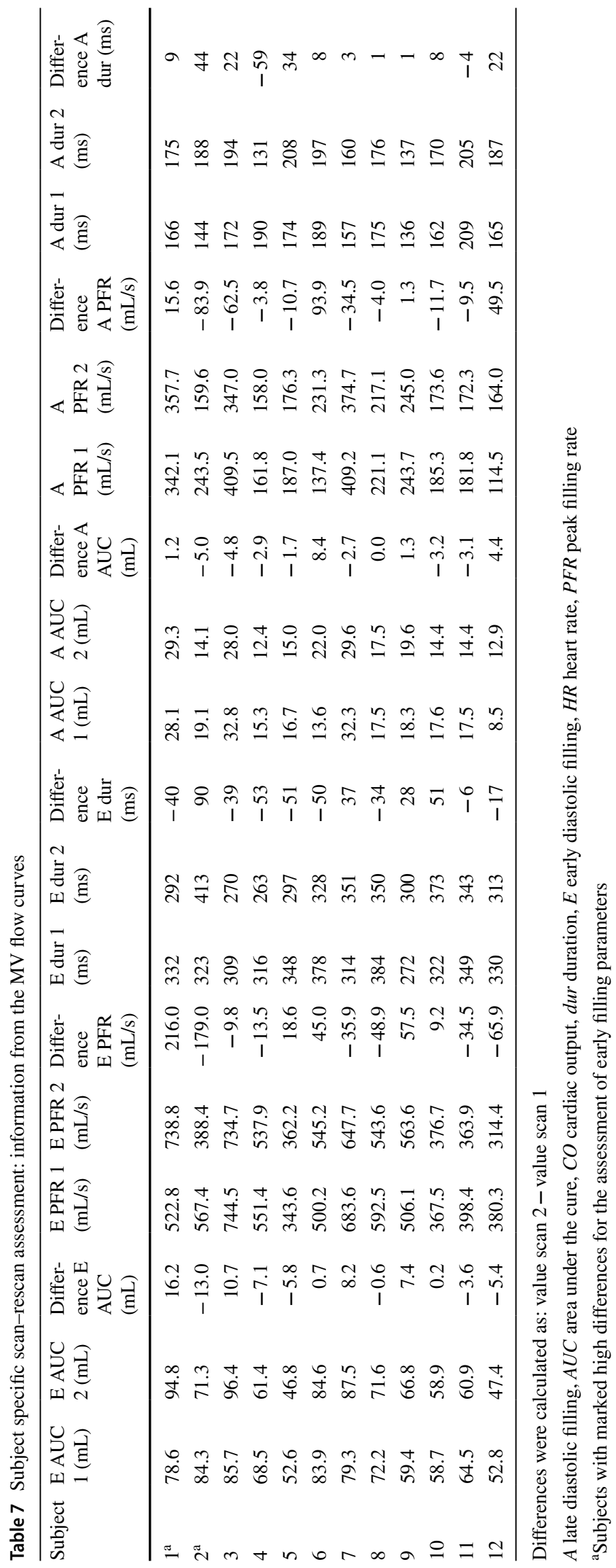




\section{References}

1. Elbaz MS, van der Geest RJ, Calkoen EE, de Roos A, Lelieveldt BP, Roest AA, Westenberg JJ (2016) Assessment of viscous energy loss and the association with three-dimensional vortex ring formation in left ventricular inflow: in vivo evaluation using fourdimensional flow MRI. Magn Reson Med. https://doi.org/10.1002/ mrm.26129

2. Pedrizzetti G, Domenichini F (2005) Nature optimizes the swirling flow in the human left ventricle. Phys Rev Lett 95(10):108101. https://doi.org/10.1103/PhysRevLett.95.108101

3. Kamphuis VP, Westenberg JJM, van der Palen RLF, Blom NA, de Roos A, van der Geest R, Elbaz MSM, Roest AAW (2017) Unravelling cardiovascular disease using four dimensional flow cardiovascular magnetic resonance. Int J Cardiovasc Imaging 33(7):1069-1081. https://doi.org/10.1007/s10554-016-1031-9

4. Eriksson J, Bolger AF, Ebbers T, Carlhall CJ (2013) Four-dimensional blood flow-specific markers of LV dysfunction in dilated cardiomyopathy. Eur Heart J Cardiov Imaging 14(5):417-424. https://doi.org/10.1093/ehjci/jes159

5. Fredriksson AG, Svalbring E, Eriksson J, Dyverfeldt P, Alehagen U, Engvall J, Ebbers T, Carlhall CJ (2016) 4D flow MRI can detect subtle right ventricular dysfunction in primary left ventricular disease. J Magn Reson Imaging 43(3):558-565. https:// doi.org/10.1002/jmri.25015

6. Kanski M, Arvidsson PM, Toger J, Borgquist R, Heiberg E, Carlsson M, Arheden H (2015) Left ventricular fluid kinetic energy time curves in heart failure from cardiovascular magnetic resonance 4D flow data. J Cardiovasc Magn Reson 17:111. https://doi. org/10.1186/s12968-015-0211-4

7. Svalbring E, Fredriksson A, Eriksson J, Dyverfeldt P, Ebbers T, Bolger AF, Engvall J, Carlhall CJ (2016) Altered diastolic flow patterns and kinetic energy in subtle left ventricular remodeling and dysfunction detected by 4D flow MRI. PLoS ONE 11(8):e0161391. https://doi.org/10.1371/journal.pone.0161391

8. Fenster BE, Browning J, Schroeder JD, Schafer M, Podgorski CA, Smyser J, Silveira LJ, Buckner JK, Hertzberg JR (2015) Vorticity is a marker of right ventricular diastolic dysfunction. Am J Physiol Heart Circ Physiol 309(6):H1087-H1093. https://doi.org/10.1152/ ajpheart.00278.2015

9. Schafer M, Browning J, Schroeder JD, Shandas R, Kheyfets VO, Buckner JK, Hunter KS, Hertzberg JR, Fenster BE (2016) Vorticity is a marker of diastolic ventricular interdependency in pulmonary hypertension. Pulm Circ 6(1):46-54. https://doi. org/10.1086/685052

10. Kheradvar AP, G (2012) Vortex formation in the heart. In: Vortex formation in the cardiovascular system. Springer, London, p 19

11. Kheradvar A, Assadi R, Falahatpisheh A, Sengupta PP (2012) Assessment of transmitral vortex formation in patients with diastolic dysfunction. J Am Soc Echocardiogr 25(2):220-227. https:// doi.org/10.1016/j.echo.2011.10.003

12. Stewart KC, Charonko JC, Niebel CL, Little WC, Vlachos PP (2012) Left ventricular vortex formation is unaffected by diastolic impairment. Am J Physiol Heart Circ Physiol 303(10):H1255H1262. https://doi.org/10.1152/ajpheart.00093.2012

13. Kamphuis VP, Roest AAW, Westenberg JJM, Elbaz MSM (2017) Biventricular vortex ring formation corresponds to regions of highest intraventricular viscous energy loss in a Fontan patient: analysis by 4D Flow MRI. Int J Cardiovasc Imaging. https://doi. org/10.1007/s10554-017-1250-8

14. Kamphuis VP, van der Palen RLF, de Koning PJH, Elbaz MSM, van der Geest RJ, de Roos A, Roest AAW, Westenberg JJM (2017) In-scan and scan-rescan assessment of LV in- and out-flow volumes by 4D flow MRI versus 2D planimetry. J Magn Reson Imaging. https://doi.org/10.1002/jmri.25792
15. Westenberg JJ, Roes SD, Ajmone Marsan N, Binnendijk NM, Doornbos J, Bax JJ, Reiber JH, de Roos A, van der Geest RJ (2008) Mitral valve and tricuspid valve blood flow: accurate quantification with 3D velocity-encoded MR imaging with retrospective valve tracking. Radiology 249(3):792-800. https://doi. org/10.1148/radiol.2492080146

16. Garg P, Westenberg JJM, van den Boogaard PJ, Swoboda PP, Aziz R, Foley JRJ, Fent GJ, Tyl FGJ, Coratella L, ElBaz MSM, van der Geest RJ, Higgins DM, Greenwood JP, Plein S (2017) Comparison of fast acquisition strategies in whole-heart four-dimensional flow cardiac MR: two-center, $1.5 \mathrm{~T}$, phantom and in vivo validation study. J Magn Reson Imaging. https://doi.org/10.1002/ jmri.25746

17. Klein S, Staring M, Murphy K, Viergever MA, Pluim JP (2010) elastix: a toolbox for intensity-based medical image registration. IEEE Trans Med Imaging 29(1):196-205. https://doi.org/10.1109/ TMI.2009.2035616

18. Agati L, Cimino S, Tonti G, Cicogna F, Petronilli V, De Luca L, Iacoboni C, Pedrizzetti G (2014) Quantitative analysis of intraventricular blood flow dynamics by echocardiographic particle image velocimetry in patients with acute myocardial infarction at different stages of left ventricular dysfunction. Eur Heart J Cardiovasc Imaging 15(11):1203-1212. https://doi.org/10.1093/ehjci/jeu106

19. Hyslop NP, White WH (2009) Estimating precision using duplicate measurements. J Air Waste Manag Assoc 59(9):1032-1039

20. Bland JM, Altman DG (1986) Statistical methods for assessing agreement between two methods of clinical measurement. Lancet 1(8476):307-310

21. Bolger AF, Heiberg E, Karlsson M, Wigstrom L, Engvall J, Sigfridsson A, Ebbers T, Kvitting JP, Carlhall CJ, Wranne B (2007) Transit of blood flow through the human left ventricle mapped by cardiovascular magnetic resonance. J Cardiovasc Magn Reson 9(5):741-747. https://doi.org/10.1080/10976640701544530

22. Mohiaddin RH (1995) Flow patterns in the dilated ischemic left ventricle studied by MR imaging with velocity vector mapping. J Magn Reson Imaging 5(5):493-498

23. Francis GS (2001) Pathophysiology of chronic heart failure. Am J Med 110(Suppl 7A):37S-46S

24. Kanski M, Toger J, Steding-Ehrenborg K, Xanthis C, Bloch KM, Heiberg E, Carlsson M, Arheden H (2015) Whole-heart fourdimensional flow can be acquired with preserved quality without respiratory gating, facilitating clinical use: a head-to-head comparison. BMC Med Imaging 15:20. https://doi.org/10.1186/ s12880-015-0061-4

25. Carlsson M, Heiberg E, Toger J, Arheden H (2012) Quantification of left and right ventricular kinetic energy using four-dimensional intracardiac magnetic resonance imaging flow measurements. Am J Physiol Heart Circ Physiol 302(4):H893-H900. https://doi. org/10.1152/ajpheart.00942.2011

26. Hussaini SF, Rutkowski DR, Roldan-Alzate A, Francois CJ (2016) Left and right ventricular kinetic energy using time-resolved versus time-average ventricular volumes. J Magn Reson Imaging. https://doi.org/10.1002/jmri.25416

27. Grothues F, Smith GC, Moon JC, Bellenger NG, Collins P, Klein HU, Pennell DJ (2002) Comparison of interstudy reproducibility of cardiovascular magnetic resonance with two-dimensional echocardiography in normal subjects and in patients with heart failure or left ventricular hypertrophy. Am J Cardiol 90(1):29-34

28. van der Geest RJ, Reiber JH (1999) Quantification in cardiac MRI. J Magn Reson Imaging 10(5):602-608 